\title{
NORMAL STATE IMPLICATURE
}

\author{
Nancy L. Green \\ Department of Computer and Information Sciences \\ University of Delaware \\ Newark, Delaware 19716, USA
}

\begin{abstract}
In the right situation, a speaker can use an unqualified indefinite description without being misunderstood. This use of language, normal state implicature, is a kind of conversational implicature, i.e. a non-truth-functional context-dependent inference based upon language users' awareness of principles of cooperative conversation. I present a convention for identifying normal state implicatures which is based upon mutual beliefs of the speaker and hearer about certain properties of the speaker's plan. A key property is the precondition that an entity playing a role in the plan must be in a normal state with respect to the plan.
\end{abstract}

\section{Introduction}

In the right situation, a speaker can use an unqualified indefinite description without being misunderstood. For example, a typical customer in a typical pet shop who said (1a) in response to the clerk's question in (1) would expect to be understood as meaning (1b). The goal of this paper is to formally describe such uses of language. ${ }^{1}$

\footnotetext{
${ }^{1}$ A similar use of language is noted in [McC87]. McCarthy (pp. 29-30) discusses the problem of bridging the gap between a "rather direct [translation] into first order logic" of a statement of the Missionaries and Cannibals puzzle, and a representation suitable for devising a solution to the puzzle. For example, if the puzzle statement mentions that "a rowboat that seats two is available" and doesn't say that anything is wrong with the boat, the problem-solver may assume that the boat doesn't leak, has oars, etc. McCarthy proposes a general-purpose method for formalizing common sense reasoning, "circumscription", to solve the problem.

Also, a similar use of language is described in [Gri75] (p. 51): "A is standing by an obviously immobilized car and is approached by $B_{i}$ the following exchange takes place:
}

A: I sm out of petrol.

$B$ : There is a garage round the corner.

... [B] implicates that the garage is, or at least may be open, [has petrol to sell], etc." That this use of language
1. (Clerk A:) May I help you?

a. (Customer B:) I'd like to see a parrot.

b. I [the speaker] would like to see a live parrot.

c. $\exists$ p:PARROT REQUEST $(B, A, \operatorname{SHOW}(A, B, p))$

d. $\exists \mathrm{q}:[\lambda$ p:PARROT LIVE(p)] REQUEST(B,A, $\operatorname{SIIOW}(A, B, q)$

One problem is that (1a) (i.e. its putative representation in (1c)) does not entail (1b) (i.e. its putative representation in (1d)). ${ }^{2}$

Another

problem is the context-dependence, both spatio-temporal and linguistic, of the relationship of (1b) to (1a). In a different spatio-temporal context, such as in a china shop, a speaker might use (1a) to convey (2) rather than (1b).

2. I [the speaker] would like to see a porcelain parrot.

In a different linguistic context, such as if the customer had said (3a) following (1a), she would not

involves the use of language I have illustrated in (1) can be seen by considering a situation identical to the above except that the dialogue consists of just A's saying "I need a garage." In other words, Grice's example is of a situation where $B$ has anticipated a request from $A$ which is the same kind of request as (1a).

${ }^{2}$ The customer's use of (1a) is an indirect speech act, namely, a request to be shown a parrot; other possible realizations of this request include "Show me a parrot" and "Can you show me a parrot?". (The derivation of representations of indirect speech acts has been treated elsewhere [PA80] and is not a concern of this paper.) (1c) is intended to represent that request by means of a first order language extended with higher-order operators such as REQUEST. Also, indefinite descriptions are represented as in [Web83]. The status of the existence of the parrot in the real world or discourse context (and the related question as to the proper scope of the existential quantifier), is not relevart to the concerns of this paper. My point is that the usuat treatments employing a one-to-one translation from surface structure to logical form without consideration of ollher information will not be able to explain the relationship of (1b) to (1a). 
normally expect the clerk to think she had meant (1b). A related question is why it would be appropriate (non-redundant) for the customer to say (3b) following (1a) if the customer believed that the clerk might mistakenly believe that the customer wanted to see a dead parrot.

\section{3.a. ... a dead one \\ b. ... a live one}

A third problem is that in order to derive (1b) from (1a) it is necessary to consider the beliefs of speaker (S) and hearer (H): e.g. S's and H's beliefs about why each said what they did, and about the appropriate state of the parrot.

Grice [Gri75] described conversational implicature, a kind of non-truth-functional contextdependent inference based upon a speaker's and hearer's awareness of principles of cooperative conversation. In this paper, I claim that a speaker's use of (1a) may conversationally implicate (1b). In order to formally describe this kind of conversational implicature, which I have termed 'normal state implicature', I adopt the methodology used by Hirschberg [Hir85] for the identification of another kind of conversational implicature, scalar implicature.

In section 2, I present a brief description of scalar implicatures and Hirschberg's methodology for identifying them. In section 3, I present a convention for identifying normal state implicar tures. Informally speaking, the convention is that if speaker $\mathrm{S}$ makes a request that hearer $\mathrm{H}$ perform an action $A$ on an entity $E$, and if $S$ and $H$ mutually believe that $S$ has a plan whose success depends on the $\mathrm{E}$ being in a certain state $\mathrm{N}$ (which is the normal state for an $\mathrm{E}$ with respect to that plan) and that S's request is a step of that plan, then $S$ is implicating a request for $S$ to do $A$ on an $E$ in state $N$.

In section 4, I clarify the notion of normal state with respect to a pian by distinguishing it from the notions of stereotype and planindependent normal state. Next, in section 5, I show how states can be represented in the lexicon. In section 6, I compare scalar and normal state implicature; in section 7, survey related work; and, in section 8 , present my conclusions.

\section{Scalar Implicature}

Hirschberg proposes the following set of six necessary and sufficient conditions for identifying conversational implicatures (p. 38) ${ }^{3}$ A speaker $S$ conversationally implicates $Q$ to a hearer $\mathbf{H}$ by saying $U$ (where $U$ is a realization of a proposition P) in a context $\mathrm{C}$ iff:

1. $S$ intends to convey $\mathrm{Q}$ to $\mathrm{H}$ via $\mathrm{U}$; and

2. $\mathrm{S}$ believes that $\mathrm{S}$ and $\mathrm{H}$ mutually believe that $\mathrm{S}$ is being cooperative; and

3. $\mathrm{S}$ and $\mathrm{H}$ mutually believe that S's saying $\mathrm{U}$ in $\mathrm{C}$, given S's cooperativity, licenses $\mathrm{Q}$; and

4. $Q$ is cancelable; i.e., it is possible to deny $Q$ without denying $P$; and

5. $Q$ is nondetachable; i.e., the choice of a realization $\mathrm{U}$ of $\mathrm{P}$ does not affect S's implicating $Q$ (except in certain situations where $Q$ is licensed via Grice's Maxim of Manner); and

6. $Q$ is reinforceable; i.e., it is possible to affirm $Q$ without seeming redundant.

Instead of using these conditions to identify particular scalar implicatures, Hirschberg argues that it is sufficient to provide a means of identifying instances of a class of conversational implicature, such as scalar implicatures. Then, she provides a convention for identifying instances of scalar implicature.

Informally speaking, scalar implicature is based on the convention that (pp. 1 - 2) "cooperative speakers will say as much as they truthfully can that is relevant to a conversational exchange"; and distinguished from other conversational implicatures by "being dependent upon the identification of some salient relation that orders a concept referred to in an utterance with other concepts"; e.g. by saying (4a), B has scalar implicated (4b). ${ }^{4}$

(4) A: How was the party last night?

a. B: Some people left early.

b. Not all people left early.

The convention for identifying scalar implicature proposed by Hirschberg is of the form: if

\footnotetext{
${ }^{3} \mathrm{Her}$ conditions are a revision of Grice's. Also, I have changed the names of her variables to be consistent with usage in the rest of my paper.

${ }^{4}(4)$ is example (1) in [Hir85].
} 
there exists a partial order $\mathrm{O}$ such that $\mathrm{S}$ and $\mathrm{H}$ mutually believe that $\mathrm{O}$ is salient in context $\mathrm{C}$, and utterance $U$ realizes the proposition that $S$ affirms/denies/is ignorant of some value in $\mathrm{O}$, then by saying $\mathrm{U}$ to $\mathrm{H}$ in $\mathrm{C}, \mathrm{S}$ licenses the scalar implicature that $\mathrm{S}$ has a particular belief regarding some other value of $\mathrm{O}$.

In the next section, I will apply Hirschberg's methodology to the problem of identifying normal state implicatures.

\section{Normal State Implicature}

In this section, I will argue that (lb) is a conversational implicature and propose a convention for identifying instances of that class of implicature, which I will call 'normal state implicature'.

First, I claim that a speaker $\mathrm{S}$ conversationally implicates (1b) to a hearer $\mathbf{H}$ by saying (1a) in the context described above; i.e. that (1b) is a conversational implicature according to the six conditions described in section 2. Condition 1 is met since $S$ intends to cause $H$ to believe (1b) by saying (1a); condition 2 since $S$ believes that it is a mutual belief of $\mathrm{S}$ and $\mathrm{H}$ that $\mathrm{S}$ is being cooperative; condition 3 will be satisfied by providing a convention for normal state implicature below. The previous discussion about (3a) and (3b) provides evidence for cancelability (condition 4) and reinforceability (condition 6), respectively; and, (1b) is nondetachable (condition 5) since alternate ways of saying (1a), in the same context, would convey (1b).

Next, in order to motivate the general convention ( $(6)$ below) for identifying normal state implicatures, I'll present the instance of the convention that accounts for the implicature in (1). Let $\mathrm{S}, \mathrm{H}, \mathrm{U}$, and $\mathrm{C}$ be constants denoting speaker, hearer, utterance, and context, respectively. Let $b_{0}, b_{1}$, and $g$ be first order variables over parrots (PARROT), live parrots (the lambda expression), and plans (PLAN), respectively. $^{5}$ HAS-PLAN(Agent,Plan,Entity) is

\footnotetext{
${ }^{5}$ The model of plans used here is that of STRIPS [FN71] with minor extensions. A plan includes preconditions which must hold in order for the plan to succeed, and a sequence of actions to be carried out to achieve some goal. One extension to this model is to add a list of entities playing a role in the plan either as instruments (e.g. a boat which is to be used to cross a river) or as the goal itself (e.g. a parrot to be acquired for a pet). The second exten-
}

true if Agent has a plan in which Entity plays a role; PRECOND(Plan,Proposition) is true if Plan has Proposition as a precondition; STEP(Plan,Action) is true if Action is a step of Plan. Also, BMB(A,B,Proposition) is true if $A$ believes that $A$ and $B$ mutually believe that Proposition; REALIZE(Utterance, Proposition) is true if Utterance expresses Proposition; REQUEST( $\mathrm{S}, \mathrm{H}_{1}$ Action) is true if $\mathrm{S}$ requests $H$ to perform Action; and $\operatorname{SAY}(\mathrm{S}, \mathrm{I}, \mathbf{U}, \mathrm{C})$ is true if $S$ says $U$ to $H$ in $C .{ }^{6} \operatorname{SHOW}(A, B, C)$ is true if A shows C to B. IN-STATE(Entity,State) is true if Entity is in the given State; and NORMAL-STATE(State,Plan,Entity) is true if State is the normal state of Entity with respect to Plan. ${ }^{7}$ Finally, NORMAL-STATEIMP(Speaker,Hearer,Utterance,Proposition,Context) is true if by use of Utterance in Context, Speaker conveys Proposition to Hearer.

Now, to paraphrase (5) below, if $S$ and $H$ mutually believe that $S$ has a plan in which a parrot plays a role and that a precondition of S's plan is that the parrot should be alive, which is its normal state with respect to the plan, and that $S$ 's saying $U$ is a step of that plan; and, if $U$ is a request to be shown a parrot, then $S$ normal state implicates a request to be shown a live parrot.

\section{5. $\forall b_{0}:$ PARROT \\ $\forall b_{1}:\left[\lambda b_{2}:\right.$ PARROT LIVE $\left.\left(b_{2}\right)\right]$ \\ $\forall g:$ PLAN \\ $\operatorname{BMB}\left(\mathrm{S}, \mathrm{H}\right.$, HAS-PLAN $\left(\mathrm{S}, \mathrm{g}, b_{0}\right) \wedge$ \\ PRECOND $\left(g, \operatorname{IN}-\operatorname{STATE}\left(b_{0}\right.\right.$, LIVE $\left.)\right) \wedge$ \\ NORMAL-STATE(LIVE, $\left.\mathrm{g}, b_{0}\right) \wedge$ \\ $\operatorname{STEP}(\mathrm{g}, \operatorname{SAY}(\mathrm{S}, \mathrm{H}, \mathrm{U}, \mathrm{C}))) \wedge$ \\ REALIZE(U, REQUEST(S, H, SHOW(H, S, $\left.\left.b_{0}\right)\right)$ ) \\ $\Leftrightarrow$ \\ NORMAL-STATE-IMP(S, H, U, REQUEST(S, H, $\left.\left.\mathrm{SHOW}\left(\mathrm{H}, \mathrm{S}, b_{1}\right)\right), \mathrm{C}\right)$}

It is possible to generalize (5) as follows. Let $\mathrm{K}, \mathrm{N}$, and $\mathrm{A}$ be higher order variables over classifications (CLASSIF), states (STATE), and actions that may be performed as a step in a plan

sion, suggested in [Car88], is to distinguish preconditions which can be achieved as subgoals from those which are unreasonable for the agent to try to bring about ("applica. bility conditions"). In (5) and (6), preconditions are meant in the sense of applicability conditions.

${ }^{8}$ BMB, REALIZE, REQUEST, and SAY are from [Hir85].

${ }^{7} \mathrm{I}$ will discuss what is meant by state and normal state in section 4. 
(ACT), respectively. Then, (6) is the general convention for identifying normal state implicature.

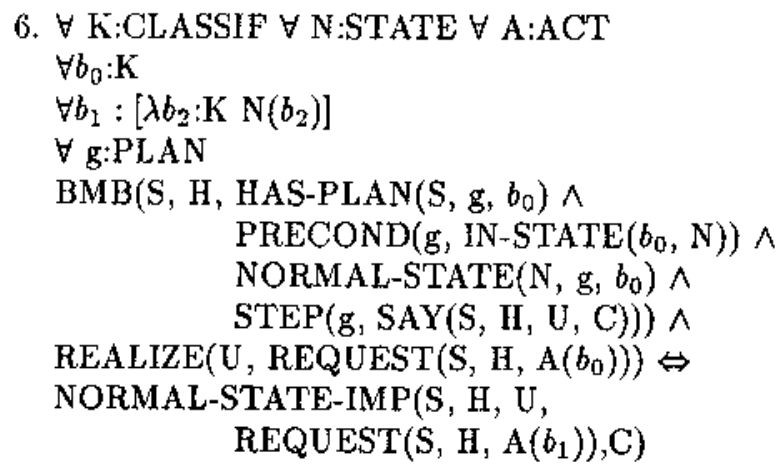

Unfortunately, if (6) is to be of maximum use, there are two problems to be solved. First, there is the problem of representing all preconditions of a plan, ${ }^{8}$ and, second, is the problem of plan inference, i.e., how does $\mathrm{H}$ come to know what S's plan is (including the problem of recognizing that the saying of $U$ is a step in S's plan)? $?^{9}$ Both problems are outside the scope of this paper.

\section{States and Normal States}

First, what I mean by a state of an entity $E$ is, adopted from [Lan87], a history of related events involving E. In Lansky's ontology, events may be causally or temporally related. Temporal precedence is transitive. Causality is not transitive and does not necessitate occurrence but does imply temporal precedence. A strong prerequisite constraint $(\rightarrow$ ) can be defined such that "each event of type $E_{2}$ can be caused by exactly one event of type $E_{1}$, and each event of type $E_{1}$ can cause at most one event of type $E_{2}$ " ([Lan87],p.142).

Many classifications expressed as nouns denote a class of entity whose state varies over the period of existence during which it is aptly characterized by the classification. For example, Figure 1 and Figure 2 depict causal event chains ${ }^{10}$ of parrots and vases, respectively.

(Nodes represent events and directed arcs represent causality.) The state of being dead or

\footnotetext{
${ }^{8} \mathrm{E}_{\text {.g., see }}[\mathrm{McC87}]$.

${ }^{9}$ E.g., see [Car88].

${ }^{10} \mathrm{I}$ don't mean 'causal chain' in the sense that philosophers have recently used it [Sch77], nor in the sense of [SA77], nor do I mean 'chain' in the mathematical sense of a total order.
}

broken can be defined in terms of the occurrence of an event type of dying or breaking, respectively. Live is the state of an entity who has been born but has not yet died; ready-to-use is the state of an artifact between its creation or repair and its destruction. ${ }^{11}$ Note that, paradoxically, language users would agree that a dead parrot or a vase with a crack in it is still aptly characterized as a parrot or vase, respectively. ${ }^{12}$

Next, what I mean by a normal state of $E$ is a state that $\mathrm{E}$ is expected to be in. For example, in the absence of information to the contrary, live or ready-to-use is expected by language users to be a state of parrots or vases, respectively. Note, however, that NORMAL-STATE in (6) represents a normal state of an entity with respect to some plan. That is, I am not claiming that, in the absence of information about S's plan, S's use of (1a) conversationally implicates (1b).

The reason for stipulating that NORMALSTATE be relative to S's plan is that use of (1a) in the context of a different plan could change what $\mathrm{S}$ and $\mathrm{H}$ consider to be normal. For example, in a taxidermist's plan, dead could be the normal state of a parrot. Also, consider 'coffee': a speaker's use of (7) in the context of a coffee farm could be used to request coffee beans; in a grocery store, a jar of instant; and in a restaurant, a hot beverage.

7. I'd like some coffee.

Note that more than one precondition of S's plan may be relevant to interpreting S's use of an expression. For example, a typical restaurant customer uttering (7) expects to be understood as not only requesting coffee in its hot-beverage state, but also in its safe-to-drink state. Also, more than one of S's plans may be relevant. Returning to the pet shop example, suppose that $\mathrm{S}$ and $\mathrm{H}$ mutually believe that $S$ has plans to acquire a parrot as a pet and also to study its vocalizations; then it would be inappropriate for $\mathbf{H}$ to show $\mathbf{S}$ a parrot that $\mathrm{H}$ believed to be incapable of making vocalizations.

Normal states differ from stereotypes. A stereotype is a generalization about prototypes of a category, ${ }^{13}$ e.g. (8). ${ }^{14}$

\footnotetext{
11 Examples of how state predicates can be defined in Lansky's formal language will be given later.

${ }^{12}$ The cracked vase example is from [Her87].

${ }^{13}$ The prototype-stereotype distinction is described in[HH83].

${ }^{14}$ Note that stereotypes may be relative to a state of the
} 
8. Unripe bananas are green.

Qualifying an expression in a way which contradicts a stereotype may have a different effect on $\mathrm{H}$ than doing so in a way which specifies a non-normal state. For instance, if $\mathrm{S}$ says (9) after saying (la) in the above pet shop scenario, H may doubt S's sincerity or S's knowledge about parrots; while S's use of (3a) after saying (1a) may cause H to have doubts about S's sincerity or H's knowledge of S's plan, but not S's knowledge about parrots.

9. ... a 100 pound one

Another difference between stereotypes and normal states is that stereotypes are not aftected by S's and H's mutual beliefs about S's plan, whereas I have just demonstrated that what is considered normal may change in the context of S's plan. Finally, another reason for making the distinction is that I am not claiming that, in the above pet shop scenario, $S$ 's use of (1a) licenses (10); i.e., $S$ does not intend to convey (10). ${ }^{15}$

10. I the speaker] would like to see a large, green, talking bird.

\section{The Role of Events in cer- tain Lexical Representa- tions}

Now I will show how the notion of state presented in the previous section can be represented in the lexicon via state predicates based on causal event chains. The purpose of this is to clarify what counts as a state and hence, what is

prototype; e.g. contrast (8) with "Ripe bananag are yellow". A statement of a stereotype in which the state of the prototypes is unspecified may describe prototypes in the plan-independent normal state for the category; e.g- consider "Bananas are yellow". Also, note that stereotypical properties may be used to convey the state; e.g. consider "I want a green banana" used to convey "I want an unripe banana".

${ }^{15}$ I recognize that it is possible for a speaker to exploit mutual beliefs about stereotypes or plan-independent normal states to convey conversational implicatures. E.g., consider the conversation: A says, "Is your neighbor rich?" $\mathrm{B}$ replies, "He's a doctor." However, this kind of implicature does not occur under the same conditions as those given for normal state implicature, and is outside of the scope of this paper. to be identified by the convention for normal state implicature. This way of representing states has benefits in other areas. First, entailment relationships between states of an entity are thereby represented. Second, certain scalar implicatures may be based on the event ordering of a causal event chain.

For example, Figure 3 contains pictorial and formal representations of a causal event chain for the ripening of fruit. Definitions of states are given as state predicates; e.g. the expression 'unripe' is used to denote a state such that no event of ripening ( $R$ ) has occurred (yet). Note that, as (11) shows, 'ripe' may be used to scalar implicate but not to entail 'not overripe'; the event ordering of the causal event chain serves as the salient order for the scalar implicature. The expected entailments follow from the constraints represented in Figure 3 .

11.a. It's ripe. In fact, it's just right for eating. b. It's ripe. In fact, it's overripe $/$ t $\infty$ ripe.

\section{Comparison of Scalar and Normal State Implicature}

These two classes of conversational implicature have some interesting similarities and differences.

First, licensing a scalar implicature requires the mention of some specific value in an ordering, while licensing a normal state implicature requires the absence of the mention of any state. For example, consider a situation where $S$ is a restaurant customer; $H$ is a waiter; $S$ and $H$ have mutual belief of the salience of an ordering such that warm precedes boiling hot; and, $\mathrm{S}$ and $\mathbf{H}$ have mutual belief of S's plan to make tea by steeping a tea bag in boiling hot water.

14.a. I'd like a pot of water.

b. I'd like a pot of warm water.

c. I'd like a pot of boiling hot water.

d. I'd like a pot of warm but not boiling hot water.

In this situation, use of (14a) would license the normal state implicature (14c) but no scalar implicature. However, use of (14b) would license the scalar implicature (14d) but not the normal state implicature (14c). (In fact, use of 'warm' in 
(14b) would cancel (14c), as well as be confusing to $\mathrm{H}$ due to its inconsistency with H's belief about S's intention to make tea.) Thus, at least in this example, scalar and normal state implicature are mutually exclusive.

Second, saliency and order relations play a role in both. Scalar implicature is based on the salience of a partially ordered set (from any domain). Normal state implicature is based on the salience of a plan; one of a plan's preconditions may involve a normal state, which can be defined in terms of a causal event chain.

\section{Related Work}

This work is related to work in several different areas.

First, one of the goals of research on nonmonotonic reasoning ${ }^{16}$ has been the use of default information. The classic example, that if something is a bird then it can fly, appears to involve all three notions that $I$ have distinguished here; namely, stereotype, plan-independent normal state, and normal state with respect to a plan. (It is a stereotype that birds are genetically suited for flight; a plan-independent normal state that a bird is alive or uninjured; and a normal state with respect to a plan to send a message via carrier $\mathrm{pi}^{-}$ geon that the bird be able to fly.) Also, I have shown that the calculation of normal state implicature is based only on the third notion, i.e., that certain "defaults" are context-dependent.

In another area, work has been done on using knowledge of a speaker's plans to fill in missing information to interpret incomplete utterances, e.g. sentence fragments [AP80] and ellipsis [Car89].

As for related work on conversational implicature, both [Hor84] and [AL81] describe pragmatic inferences where what is conveyed by an utterance is more precise than its literal meaning. They claim that such inferences are based on a principle of speaker economy and exploit the speaker's and hearer's shared beliefs about stereotypes. Also, Horn points out that an unmarked expression tends to be associated with the stereotype of an extension and its marked counterpart with the non-stereotype. Roughly, this corresponds to my observation regarding (14), that the absence of a qualification (the unmarked case) licenses a

\footnotetext{
${ }^{16}$ For a survey, see [Gin87].
}

normal state implicature, while the presence of a qualification (the marked case), blocks it (thereby allowing the scalar implicature to be conveyed).

Finally, Herskovits [IIer87] addresses the problem that the meaning of a locative expression varies with the context of its use. Her approach is to specify "a set of characteristic constraints constraints that must hold for the expression to be used truly and appropriately under normal conditions. " (p. 20) Her constraints appear to include stereotypes and plan-independent normal states; normal is distinguished from prototypical; and the constraints may include speaker purpose.

\section{Conclusions}

This paper has provided a convention for identifying normal state implicatures. Normal state implicature permits a speaker to omit certain information from an indefinite description in certain situations without being misunderstood. The convention is that if $S$ makes a request that $\mathrm{H}$ perform an action $A$ on an $\mathrm{E}$, and if $\mathrm{S}$ and $\mathrm{H}$ mutually believe that $\mathrm{S}$ has a plan whose success depends upon the $\mathrm{E}$ being in the normal state $\mathrm{N}$ with respect to that plan, and that S's request is a step of that plan, then $S$ is implicating a request for $S$ to do $\mathrm{A}$ on an $\mathrm{E}$ in state $\mathrm{N}$.

In order to specify the convention for normal state implicature, I distinguished the notions of stereotype, plan-independent normal state, and normal state with respect to a plan. This distinction may prove useful in solving other problems in the description of how language is used. Also, a representation for states, in terms of causal event chains, was proposed.

The convention I have provided is important both in natural language generation and interpretation. In generation, a system needs to consider what normal state implicatures would be licensed by its use of an indefinite description. These implicatures determine what qualifications may be omitted (namely, those which would be implicated) and what ones are required (those which are needed to block implicatures that the system does not wish to convey). ${ }^{17}$ In interpretation, a system may need to understand what a user has

\footnotetext{
${ }^{17}$ This latter behavior is an example of Joshi's revised Maxim of Quality: "If you, the speaker, plan to say any+ thing which may imply for the hearer something you believe to be false, then provide further information to block it." [Jos82]
} 
inplicated in order to provide a cooperative response. For instance, if during a dialogue a system has inferred that a user has a plan to make an immediate delivery, and then the user says (15a), then if the system knows that the only truck in terminal $\mathbf{A}$ is out of service, it would be uncooperative for the system to reply with (15b) alone; (15c) should be added for a more cooperative response.

15.a. User: Is there a truck in terminal A?

b. System: Yes, there is one

c. but it's out of service.

This work may be extended in at least two ways. First, it would be interesting to investigate what plan inference algorithms are necessary in order to recognize normal state implicatures in actual dialogue. Another question is whether the notion of normal state implicature can be generalized to account for other uses of language.

\section{Acknowledgments}

An earlier version of this work was done at the University of Pennsylvania, partially supported by DARPA grant N00014-85-K0018. My thanks to the people there, particularly Bonnie Webber and Ellen Prince. Thanks to my colleagues at SAS Institute Inc., Cary, N. C., for their moral support while much of this paper was being written. The final draft was prepared at the University of Delaware; thanks to the people there, especially Sandra Carberry and K. Vijayashanker.

\section{References}

[AL81] Jay David Atlas and Stephen C. Levinson. It-clefts, informativeness, and logical form: radical pragmatics (revised standard version). In Peter Cole, editor, Radical Pragmatics, pages 1-62, Academic Press, N. Y., 1981.

[AP80] James F. Allen and C. Raymond Perrault. Analyzing intention in utterances. Artificial Intelligence, 15:143-178, 1980.

[Car88] Sandra Carberry. Modeling the user's plans and goals. Computational Linguistics, 14(3):23-37, 1988.
[Car89] Sandra Carberry. A pragmatics-based approach to ellipsis resolution. Computational Linguistics, 15(2):75-96, 1989.

[FN71] R. E. Fikes and N. J. Nilsson. Strips: a new approach to the application of theorem proving to problem solving. Artificial Intelligence, 2:189-208, 1971.

[Gin87] Matthew L. Ginsberg. Readings in Nonmonotonic Reasoning. Morgan Kaufmann, Los Altos, California, 1987.

[Gri75] H. Paul Grice. Logic and conversation. In P. Cole and J. L. Morgan, editors, Syntax and Semantics III: Speech Acts, pages 41-58, Academic Press, N.Y., 1975.

[Her87] Annette Herskovits. Language and Spatial Cognition. Cambridge University Press, Cambridge, England, 1987.

[HH83] J. Hurford and B. Heasley. Semantics: A Coursebook. Cambridge University Press, Cambridge, England, 1983.

[Hir85] Julia Bell Hirschberg. A Theory of Scalar Implicature. Technical Report MS-CIS-85-56, Department of Computer and Information Science, University of Pennsylvania, 1985.

[Hor 84] Larry Horn. Toward a new taxonomy for pragmatic inference: q-based and $\mathbf{r -}$ based implicature. In D. Schiffrin, editor, GURT '84. Meaning, Form and Use in Context: Linguistic Applications, pages 11-42, Georgetown University Press, Washington, D. C., 1984.

[Jos82] Aravind K. Joshi. Mutual beliefs in question-answer systems. In N. Smith, editor, Mutual Beliefs, pages 181-197, Academic Press, New York, 1982.

[Lan87] Amy Lansky. A representation of parallel activity based on events, structure, and causality. In M. P. Georgeff and A. Lansky, editors, Reasoning about Actions and Plans: Proceedings of the 1986 Workshop, pages 123-160, Morgan Kaufmann, 1987.

[McC87] John McCarthy. Circumscription - a form of non-monotonic reasoning. In Matthew L. Ginsberg, editor, Readings in Nonmonotonic Reasoning, pages 145- 
[PA80] R. Perrault and J. Allen. A plan-based analysis of indirect speech acts. Amer. ican Journal of Computational Linguistics, 6(3-4):167-182, 1980.

[SA77] Roger C. Schank and Robert P. Abelson. Scripts, Plans, Goals and Under. standing. Lawrence Erlbaum Associates, Hinsdale, New Jersey, 1977.

[Sch77] Stephen P. Schwartz. Introduction. In Stephen P. Schwartz, editor, Naming, Necessity, and Natural Kinds, pages 1341, Cornell University Press, 1977.

[Web83] Bonnie L. Webber. So what can we talk about now? In Jones K. S. Grosz, B. and B. L. Webber, editors, Readings in Natural Language Processing, Morgan Kaufmann, Los Altos, California, 1983.

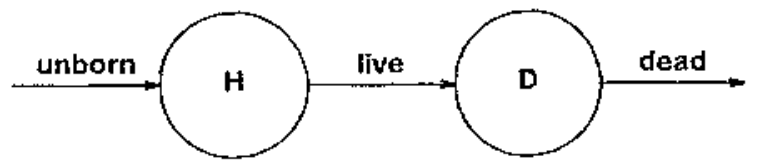

Figure 1: Causal event chain for parrot

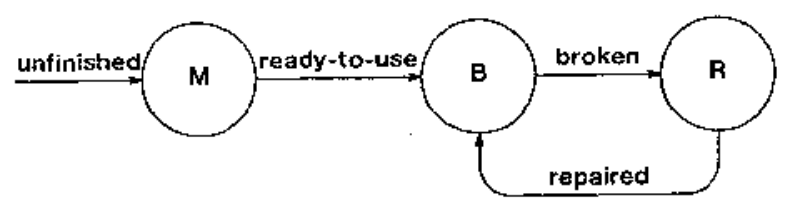

Figure 2: Causal event chain for vase

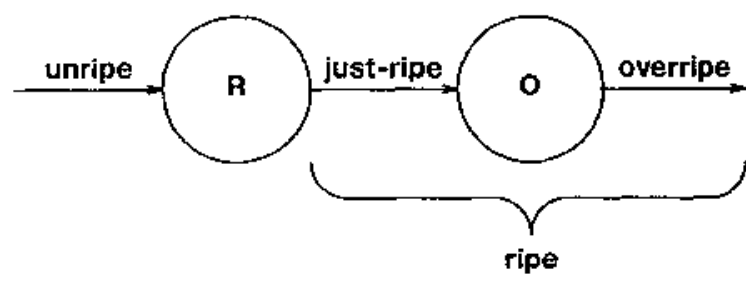

Fruit-for-eating = element type events

R [Ripen]

O [Become Overripe]

constraints

1. $\mathrm{R} \rightarrow \mathrm{O}$

end element type

unripe $(x) \equiv \neg(\exists$ r:x.R) occurred(r)

just-ripe $(x) \equiv(\exists \mathrm{r}: \mathrm{x} \cdot \mathrm{R}) \operatorname{accurred}(\mathrm{r}) \wedge$

$\neg((\exists \circ x .0)$ occurred $(0) \wedge r \rightarrow 0)$

overripe $(x) \equiv(\exists$ o:x.O) occurred(o)

ripe $(\mathrm{x}) \equiv(\exists \mathrm{r}: \mathrm{x} . \mathrm{R})$ occurred(r)

Figure 3: Causal event chain for fruit ripening 